\title{
Brief introduction to pharmaceutical microbiologist
}

\section{Introduction}

The aim of this article is that everyone either is the scientific field or not in it, to be familiar with the microbiology generally and specially with role of QC Pharmaceutical Microbiologist, for that the article is divided into five parts: the first is a brief introduction about Microbiology, second is a brief introduction about who is the Microbiologist and what he do, third is an introduction to Microbiology laboratory and what it contains of equipments and instruments, the fourth about Pharmaceutical plant and their classifications, and the fifth is about the role of QC Pharmaceutical Microbiologist.

\section{Microbiology}

Microbiology is the study of microscopic living organisms, such as bacteria and fungi. The branches of microbiology can be classified into pure and applied sciences. Microbiology can be also classified based on taxonomy Pure Microbiology encompasses numerous subdisciplines including, mycology (study of Fungi), bacteriology (study of bacteria) and Virology (study of viruses).

There are many different types of applied microbiology. Medical microbiology is perhaps the most well-known because it deals with the roles that microbes have in human illness. Other types include environmental microbiology, food microbiology and pharmaceutical microbiology. All these deal with the way microbes or microorganisms affect the environment, the food supply and the health care industry. There is considerable overlap between the specific branches of microbiology with each other and with other disciplines, and certain aspects of these branches can extend beyond the traditional scope of microbiology.

\section{Microbiologist}

A microbiologist is usually a person with a minimum of bachelor's degree in Microbiology or a related field such as biology, chemistry. Microbiologist usually works in hospitals/clinical laboratories, food industry, environment, research laboratories, pharmaceutical industry. The role of the microbiologist is to investigate the growth and characteristics of microscopic organisms such as bacteria, algae or fungi.

\section{Microbiology laboratory}

Microbiology lab is the place where all microbiological tests and analysis occur, it have special preparations in design and precautions as the hazard material here is Microorganisms-which might be infectious- which is different from chemical and physical lab as here these pathogens can multiple or transfer out of the lab if precaution is not followed. The design of the lab have to ensure that the floor, walls and benches it the lab doesn't support or permit Microorganisms growth. The scientist working is the microbiology lab is always safe as long as they follow precautions and are focused in their work. Different equipments and instruments is used in microbiology lab, we will list some of basic devices needed in the Microbiology laboratory.

\section{Autoclave}

It's a sterilization device that is used to sterilize media; tools used
Volume I Issue 5 - 2014

\author{
Mohamed Magdy \\ Department of Microbiology, International Drug Agency for \\ Pharmaceutical Industry (IDI), Egypt
}

Correspondence: Mohamed Magdy, Department of Microbiology, International Drug Agency for Pharmaceutical Industry (IDI), Egypt, Tel +20I006222495,

Emailm_Magdy_85@hotmail.com

Received: October 25, 2014 | Published: October 29, 2014

in microbiology lab, or decontaminating biohazard wastes. It sterilize by using High temperature and pressure.

\section{Incubators}

It's a device used to maintain the growth of Microorganisms through controlling temperature, humidity or other factors which is essential for the growth of certain types of Microorganisms.

\section{Laminar flow cabinet}

It's a carefully enclosed bench designed to prevent contamination of microbiological samples. Where air is drawn through a HEPA filter and blown in a very smooth, laminar flow towards the user. The cabinet is usually made of stainless steel with no gaps or joints where spores might collect.

\section{Refrigerator}

Used in storing media under low temperatures to prevent microorganisms' growth and to avoid dehydration of the media.

\section{Microscope}

Is an instrument used to see Bacteria and fungi for purpose of identification and characterization.

\section{Chemicals}

Different types of chemicals is found in the microbiology laboratory but most type can be found in the microbiology is the Microbiological media which is used as nutrient for the growth, enrichment and identification of microorganisms, other types of chemicals can be found mainly also for the identification of microorganisms.

\section{Pharmaceutical plants}

Pharmaceuticals plants are divided mainly into two categories:

Sterile: where the final products are sterile (free of microorganisms) such as eye drops and parenteral.

Non sterile: where the final product contain Microorganisms within defined limit such as tablets and capsules.

The type of the final product affects the design of the planet as each type of them require special limits in construction, air particle size, microorganisms numbers in air and water. 
For that the pharmaceutical plants must have controlled Environment with specific limits depending on the product it produce , also water used in Pharmaceutical industry must be purified.

Pharmaceutical plants have to follow guidance of GMP (good manufacturing practice).

\section{Role of QC pharmaceutical microbiologist}

A Microbiologist in a pharmaceutical factory is a member of Quality department, the role of the Microbiologist is to ensure the quality of raw materials before they are processed in the production area, monitor the microbiological quality of Environment (air monitoring) and water, and validate the test methods used in testing finished products, and test finished products from Microbiological prospective. For the Microbiologist to perform his/her duties some devices have to found in the lab as already mentioned, also the microbiologist has to prepare media and diluents and calibrate equipments used on the lab based on their SOP (standard operating procedure). Precautions have to be always followed in the Microbiology lab and wearing laboratory uniform and laboratory safety clothes (lab Coat, gloves, overhead) We will discuss here some of the main tests performed in non sterile pharmaceutical Microbiology lab:

\section{Enumeration test of the finished products and raw materials}

Which is referred as TAMC (Total Aerobic Microbial Count) and TYMC (total combined yeast and Mould), the purpose of these tests is to find the number of bacteria and fungi found in the tested material and whether it is in the limit or not, the limit is defined from USP (United States Pharmacopeia), EP (European pharmacopeia), BP (British Pharmacopeia) or the pharmacopeia used in the country of the lab. There are many types of tests to find the TAMC and TYMC of a tested material we will go through them briefly now, most used test is Pour plate method where a sample of the tested material is added to a sterile Petri dish and then the media is added, the plates is incubated and the growth is examined after the incubation period.

Another method is filtration method where the tested product is filtered through a sterile filter then the filter is added to media and incubated and examined after the incubation period ,but the method is not always applicable as some materials is not filterable.

The third method is called MPN (most probable number) and it's the least accurate of the there and it's only used when the other two types is not applicable. Where the sample is diluted in 3 tubes of 3 consecutive concentrations and incubated, then the tubes is examined for turbidity and the result of the test is known using MPN table.

\section{Test for specified microorganisms}

These tests is used to determine the absence or limited occurrence of specified Microorganisms, each microorganisms has his own biological properties such as producing certain enzyme, for that reason certain media is used to detected specified Microorganisms where these media contain chemicals which interact the enzyme produced by the Microorganisms such as using Macconkey agar bacteria to detect E.coli where E.coli have characteristic amoeboid shape on the media and capable of precipitating bile salts in the media. Microorganisms which are detected in USP \& BP are Pseudomonas Aeruginosa, Staphylococcus Areus, Ecshericha Coli, Salmonella Spp., and Bile tolerant gram negative bacteria.

\section{Environmental monitoring}

Where the purpose of these tests to find the number of Microorganisms found in the air in the production areas or in the lab during work and whether they are in the Limit or not.

Environmental Monitoring is divided into:

Active monitoring: Where an Air sampler is used which a device is used to collect a predetermined volume of air and pass it on Petri dish containing media then the Petri dish is incubated and examined after the incubation period.

Passive monitoring: Where Settled plate Method is used where Petri dished Containing media is left open in certain places for certain period of time then closed and transferred in the incubator, and examined after the incubation period.

Personnel monitoring: Where sterile swab is used to swab the gloves of the personnel to find any possible microbiological contaminant that can be acquired to the product from the personnel.

Walls and floors monitoring: Where Rodac plates is used which have a raised convex media which become in contact with the examined wall or floor then it's also incubated and examined after incubation period.

Other tests is done in the Microbiology laboratory but these are considered the main tests in non-sterile plants, others test can be done to ensure the quality of the media used as Growth promotion test, or test to validate the test method of the Finished product or raw materials, tests for Enumeration of TAMC in the purified water, and detection of specified microorganisms in the water. A lot of articles and researches is done to cover Pharmaceutical Microbiology but I hope here that you had a brief useful introduction to Pharmaceutical World.

\section{Acknowledgments}

None.

\section{Conflicts of interest}

Author declares that there is no conflict of interest. 